\title{
A Self-tuning Controller for Real-time Voltage Regulation
}

\author{
Weiming Li, Xiao-Hua Yu
}

\begin{abstract}
In this research, a self-tuning controller based on multi-layer feed-forward neural network is developed for realtime output voltage regulation of a class of DC power supplies. The neural network based controller has the advantage of adaptive learning ability, and can work under the situations when the input voltage and load current fluctuate. LevenbergMarquardt back-propagation training algorithm is used in computer simulation. The neural network controller is implemented and tested on hardware using a DSP (digital signal processor). Experimental results show that this neural network based approach outperforms the conventional analog controller, in terms of both line regulation and load regulation.
\end{abstract}

\section{INTRODUCTION}

$\mathrm{N}$ OWADAYS DC-DC converters can be found in almost every electronic device, since all the semiconductor components are powered by DC source. PWM (pulse-width modulation) technique is used in many voltage regulators. It changes the average value (i.e., dc component) of a square waveform by modulating its duty cycle.

One of the design objectives for electronic engineers is to improve the efficiency of power conversion. In PWM converters, a switching network is employed for square waveform modulation. Ideally, the power dissipated by the switch network is zero; however in practice, the power efficiency of a typical DC-DC converter could be as low as $70 \%$. Many different kinds of topologies ([1], [2], [3]) have been investigated in the past to reduce the switching loss. Unfortunately, those topologies either need additional components for the power circuit, which may introduce some unstable factors to the circuit; or operate at variable frequency, which makes the filter design at output stage very difficult. Phase-shifted zero-voltage switching full-bridge converters overcome the above problems and thus have been received more and more attention recently. It employs zerovoltage-switching (ZVS) technique which allows the voltages across the transistors to swing to zero just before the start of the next conduction cycle ([4], [5], [6]).

The conventional approach assumes that the circuit is operated around its equilibrium state, and then derives a set

This work was supported in part by the Department of the Navy, Office of Naval Research, under Award \# N00014-05-1-0855.

Weiming $\mathrm{Li}$ was with the Department of Electrical Engineering, California Polytechnic State University, San Luis Obispo, USA. He is now with St. Jude Medical, Sylmar, CA, USA.

Xiao-Hua $\mathrm{Yu}$ is with the Department of Electrical Engineering, California Polytechnic State University, San Luis Obispo, CA 93407, USA (e-mail: xhyu@ee.calpoly.edu). of linear equations based on this assumption [9] [10]. However, in practice, the supply voltage and load current may have a wide range of variation; so the controller has to be designed to work under such conditions.

Artificial neural networks (ANN) have been widely used in the field of system identification, adaptive control, and statistic modeling in recent years. A neural network is composed of many non-linear adaptive processing elements and is capable of approximating any measurable function under certain conditions.

Recently, artificial neural network based controller has been chosen as an alternative to classic methods ([7], [8]) to improve the performance of DC power supply to dynamical system changes, due to the adaptive learning ability of the neural network controller. However, no prior work has been done to control a PSFB (Phase-Shifted Full-Bridge) converter using the neural network approach yet. In this research, an approach based on multi-layer feed-forward neural network controller is investigated. A Matlab Simulink model is developed first to generate the data set; then the neural network is trained by Levenberg-Marquardt back-propagation algorithm. Finally, the neural controller is implemented on a DSP evaluation board eZdsp ${ }^{\circledR}$ F2812 (with digital processor TMS320F2812) and a phase-shifted zero-voltage-switching circuit board UCC3895EVM. Satisfactory experimental results with the neural network controller are obtained and compared with the conventional analog controller, in terms of both line regulation and load regulation.

\section{Modeling AND SIMULATION}

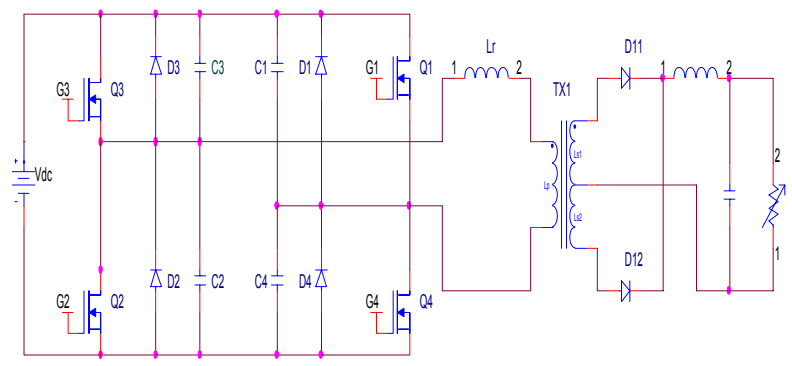

Fig 1. PSFB DC-DC Converter

The circuit diagram of a PSFB (Phase-Shifted FullBridge) DC-DC converter is shown in Fig. 1. Based on circuit analysis, the control scheme to drive the switching MOSFET (Q1, Q2, Q3 and Q4) is very complicated [6]. The circuit is operated in one of the following modes: 
Mode 1: The diagonal MOSFET Q3 \& Q4 are conducting and power is delivered through the transformer to the load. The primary load current is flowing through the leakage inductance of the transformer. The total primary current is equal to the load current plus increasing magnetizing current of the transformer.

Mode 2: With Q4 on, the capacitance across Q1 is charged to $+\mathrm{V}$. When Q4 turns OFF the current through the transformer inductance starts to charge the drain source capacitance of Q4, while at the same time discharges the capacitance of Q1. This action continues until the body diode of Q1 turns ON to clamp the voltage across Q1 at approximately $0.7 \mathrm{~V}$. The current through the transformer is sustained in the upper half of the power circuit.

Mode 3: When the voltage across Q1 reaches approximately $0 \mathrm{~V}, \mathrm{Q} 1$ turns $\mathrm{ON}$. The time that is required for the capacitance of Q4 and Q1 to reach the desired voltage is determined by the characteristics of hardware. The current in this mode circulates through the conduction channels of Q3 and Q1.

Mode 4: Q3 turns off. The transformer current now starts to charge and discharge the capacitance of Q3 and Q2 respectively. It again requires a finite amount of time for the drain voltage of Q2 to reach "0 volt" at which point Q2 is allowed to be switched on. That means non-dissipate turn ON switching is accomplished.

Mode 5: With the complete discharge of its drain source capacitance Q2 is now ready to turn ON. Power is delivered to the load through the conduction path of Q1 and Q2 for an amount of time that is determined by the control circuit. Twice the product of this time, times the operating frequency of the oscillator gives the duty cycle of the converter as in a regular PWM converter; i.e., duty cycle $D=2 \cdot T_{\text {on }} \cdot F_{s}$.

Mode 6: Following the power transfer by the above diagonal pair, Q1 turns OFF. The voltage across Q4 starts to decrease, and when this voltage reaches 0 volts the next mode starts.

Mode 7: In this mode, Q4 turns on and primary current circulates in the conduction channels of the lower pair.

Mode 8: Q2 turns OFF and current starts to charge and discharge the capacitance of Q2 and Q3 respectively. When the voltage across Q3 has reached 0 volts then Q3 turns ON non-dissipative and the complete cycle repeats itself from Mode 1.

The relationship between the input/output voltage and the duty cycle can be described as:

$$
V_{0}=\left(\frac{2 n V_{i n}}{T_{s}}\right)\left(\Phi \cdot \frac{T_{s}}{2}-T_{1}-\frac{T_{2}}{2}\right)
$$

where $V_{0}$ is the output voltage, $V_{\text {in }}$ is the input voltage, both in RMS value; $\mathrm{n}$ is the transformer ratio, $T_{s}$ is the switching period constant, and $\Phi$ is the duty cycle. $T_{1}$ and $T_{2}$ can be obtained from the following equations [11]:

$$
\begin{aligned}
& T_{1}=\frac{\left(n I+I_{c}\right) L_{r}}{V_{i n}} \\
& T_{2}=\frac{V_{i n}\left(C_{Q 3}+C_{Q 4}\right)}{n I}
\end{aligned}
$$

where $I$ is the load current; $C_{Q 3}$ and $C_{Q 4}$ are the capacitance of the two intrinsic capacitors of Q3 and Q4, respectively; $L_{r}$ is the resonant inductance. $I_{c}$ can be calculated as follows:

$$
I_{c}=V_{\text {in }} \sqrt{\frac{\left(C_{Q 1}+C_{Q 2}\right)\left(J^{2}-1\right)}{L_{r}}}
$$

where

$$
J=\frac{n I}{V_{i n}} \sqrt{\frac{L_{r}}{\left(C_{Q 1}+C_{Q 2}\right)}}
$$

The controller determines the duty cycle based on the input voltage and load current to achieve the desired output voltage. As shown in the above equations, the control law is highly nonlinear and as the result, it leads to a very complicated design for conventional approach.

Based on the above the analysis, the Simulink model for training the neural network controller is developed. It is well-known that artificial neural networks can approximate any nonlinear function to the desired accuracy, after it is fully trained. A feedforward neural network with six neurons in the hidden layer and one neuron in the output layer is employed. The activation function for each neuron is:

$$
f(x)=\frac{1}{1+e^{-x}}
$$

The weights of the neural network are initialized at random, and then updated by back-propagation algorithm. To speed up the training, Levenberg-Marquardt algorithm is employed:

$$
W(k+1)=W(k)+\Delta W
$$

where

$$
\Delta W=\left(J_{a}{ }^{T} J_{a}+\mu I\right)^{-1} J_{a}{ }^{T} e
$$

where $J_{a}$ is the first order derivative of the error function respect to the neural network weight (also called the Jacobian matrix), e is the output error (i.e., the difference between the neural network output and the desired output), $\mu$ is a learning parameter, and $\mathrm{k}$ is the number of iterations.

The computer simulation results are shown in the following figures. Fig. 2 shows the training error is reduced to the desired accuracy after about 100 iterations (the percentage of RMS error is about $0.01 \%$ ). Fig. 3 demonstrates the duty cycle estimation of the neural network 
and the desired value, where "plant" is the desired value of duty cycle while "network" is the output of the neural network. It is shown that once the neural network is trained, it is able to change the duty cycle based on the change of the output voltage.

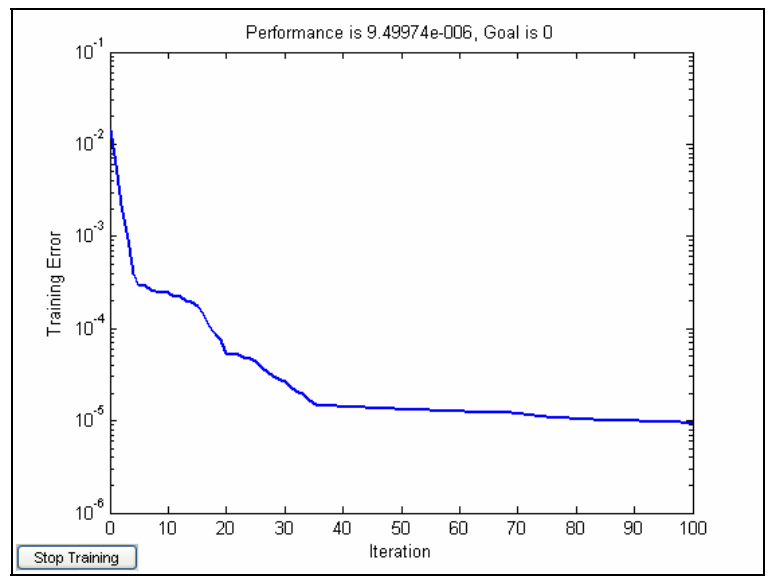

Fig 2. Neural Network Training Error

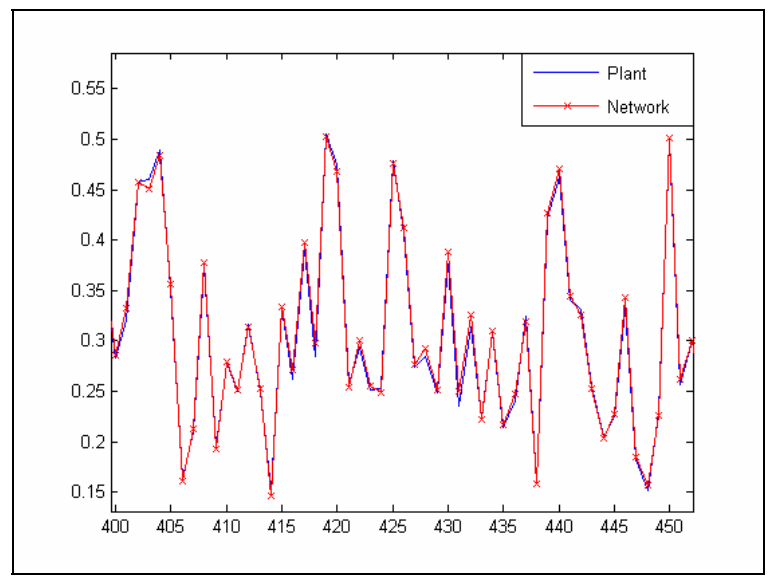

Fig. 3. Duty Cycle Estimation

\section{EXPERIMENTAL RESULTS}

The overall system block diagram is shown in Fig. 4. The inputs to the neural network include input voltage, load current, and the change of output voltage. The control objective is to keep the output voltage stable (at the nominal value of $3.3 \mathrm{~V}$ in this case) under the conditions of different input voltages and load currents.

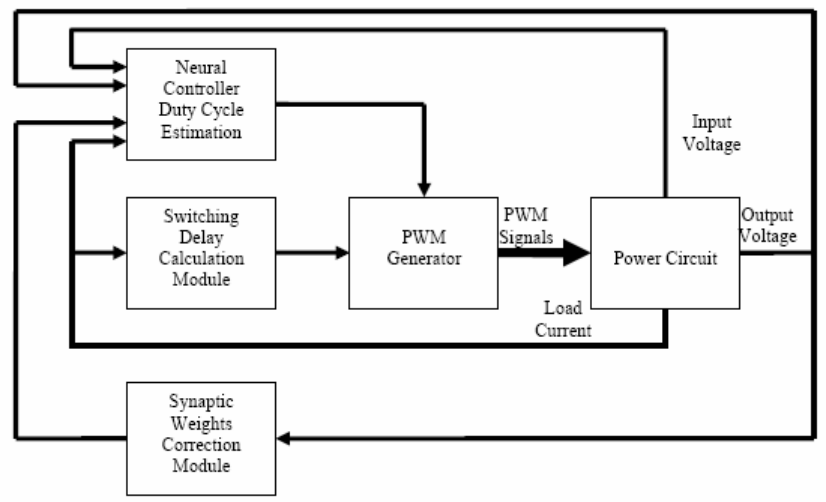

Fig. 4. The System Block Diagram

In order to investigate the performance of the neural network controller from experimental data, a DSP (Digital Signal Processor) evaluation module eZdsp ${ }^{\circledR}$ F2812 and a phase-shifted zero-voltage-switching evaluation board UCC3895EVM are used to implement the neural controller and the power circuit, respectively. The on-board digital signal processor TMS320F2812 is a 32-bit CPU with 150 MIPS (million instructions per second) operating speed. The phase-shifted full-bridge converter is operated on $48 \mathrm{~V}$ (nominal input) and it provides an output of $3.3 \mathrm{~V}$. The neural controller is developed in $\mathrm{C}$ language. In Fig. 4, the PWM generator and the power circuit blocks are implemented on board UCC3895EVM and the rest of the blocks (i.e., the neural network controller and the switching delay calculation module) are implemented with DSP TMS320F2812.

First, the analog signals from the power circuit are digitized and processed before being fed into the neural network's inputs. Since the power circuit is driven by 400 $\mathrm{kHz}$ high frequency PWM signals, the raw output voltage signal is quite noisy during the switch turn-on and turn-off time. To solve this problem, multiple samples are taken to obtain the average value over a certain period of time. Next, the processed data is fed into the neural network to estimate the desired duty cycle. The new estimated duty cycle is then applied to the power circuit, and the output voltage is monitored. The computational flow chart is shown in Fig. 5.

At nominal input voltage $48 \mathrm{~V}$, the converter's output voltage in steady-state is measured under different load conditions and the result is shown in Figure 6, where the curve on top is the output controlled by neural network controller and the other one is the output controlled by a conventional analog controller (UCC3895). The desired nominal output voltage is $3.3 \mathrm{~V}$ by design. As shown in the plot, the maximum output (by the neural network controller) is $3.38 \mathrm{~V}$, which results in only $2.4 \%$ error rate. The overall neural network controller outperforms the conventional analog controller. 


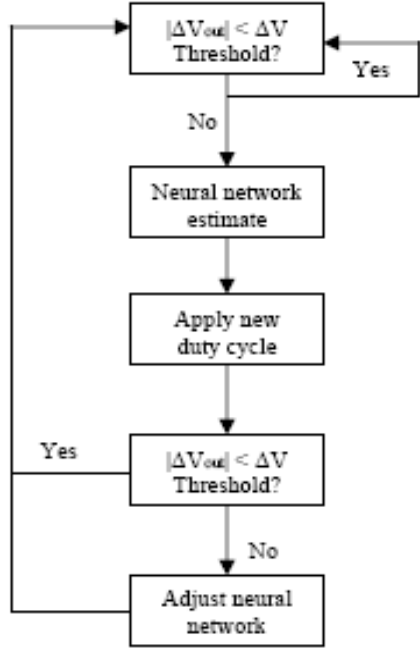

Fig. 5. The Flow Chart

The percentage of load regulation gives us an indication of how much the output voltage changes over a range of load resistance values. In constant voltage mode, variations in the load result in changes in the load current. Here, we define the percentage of load regulation between $0 \%$ and $100 \%$ of the full load as:

$$
\text { Load_Re } g=\frac{\left|V_{0(\text { noload })}-V_{0(\text { full load })}\right|}{V_{0(\text { nominal })}} \times 100 \%
$$

where "Load_Reg" represents the percentage of line regulation, $V_{0 \text { (noload) }}$ represents the output voltage when the load current is 0 , and $V_{0 \text { (full load) }}$ represents the output voltage when full load is presented, and $V_{0 \text { (nominal })}$ represents the ideal (nominal) output voltage. Experimental results show that the percentage of load regulation is only about $2.1 \%$, which concludes that the neural controller provides excellent load regulation (comparing to the load regulation of $4.5 \%$ using the conventional controller).

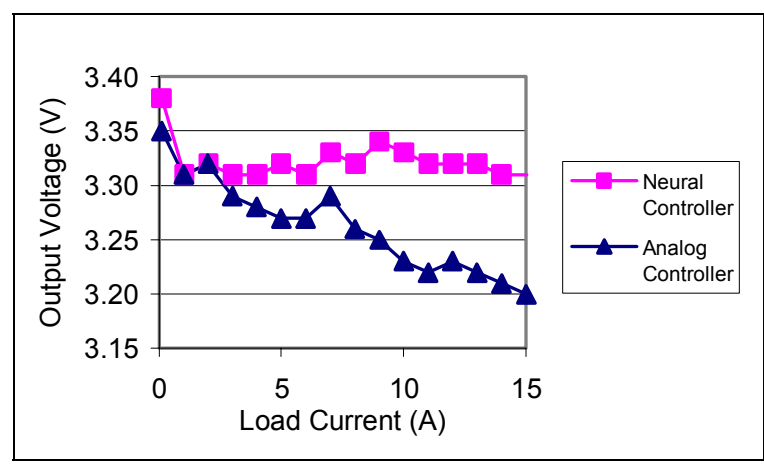

Fig. 6. Load Regulation at 48V Nominal Input Voltage

At full load condition, the converter's output voltage is measured at different input voltages, and the result is shown in Fig. 7. The maximum output error is only $0.04 \mathrm{~V}$, which is less than $2 \%$ of the nominal value. Another performance measure, the percentage of line regulation between $48 \mathrm{~V}$ and $72 \mathrm{~V}$ input voltage (i.e., "Line_Reg") is calculated using the following formula:

$$
\text { Line_Reg }=\frac{\left|V_{0\left(h i \_i n\right)}-V_{0\left(l o \_i n\right)}\right|}{V_{0(\text { nominal })}} \times 100 \%
$$

where $V_{0\left(h i_{i} \text { in }\right)}$ is the output voltage under the highest input voltage which can be allowed ( $72 \mathrm{~V}$ in this case), and $V_{0\left(l o \_i n\right)}$ is the output voltage with the lowest input voltage ( $48 \mathrm{~V}$ in this case). The neural controller achieves better line regulation $(0.9 \%)$ than the analog controller $(3.9 \%)$.

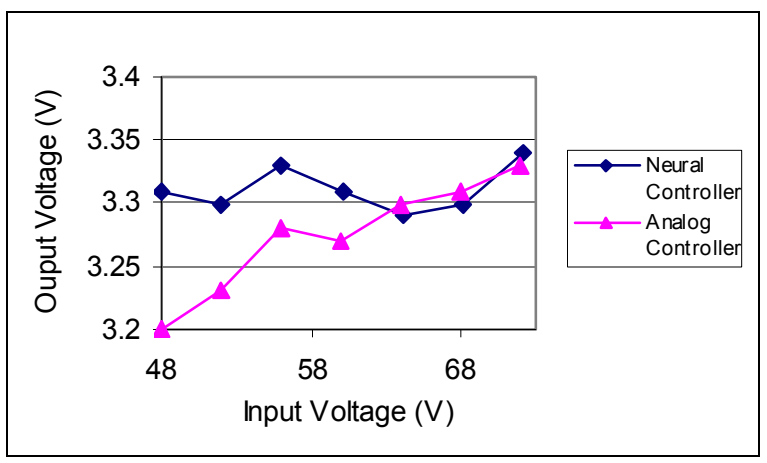

Fig. 7. Line Regulation at Full Load 15A

\section{CONCLUSION}

A neural controller as an alternative to classic controller for PSFB DC-DC converter is proposed in this paper. Both simulation and experimental results show that the neural network controller is able to estimate the desired duty cycle under several dynamic conditions. In terms of both line regulation and load regulation, the neural network based approach outperforms the conventional analog controller. More research works will be done to optimize the software to speed up the neural controller for real-time applications.

\section{ACKNOWLEDGEMENT}

The authors would like to thank Dr. Taufik for providing the hardware.

\section{REFERENCES}

[1] P. R. Chetty, "Resonant power supplies: Their history and status," IEEE Aerosp. Electron. Syst. Mag., vol. 7, no. 4, pp. 23-29, Apr. 1992.

[2] M. G. Kim and M. J. Youn, "An energy feedback control of series resonant converters," IEEE Trans. Power Electron., vol. 6, no. 4, pp. 338-345, Jul. 1991.

[3] J. M. Carrasco, E. Galván, G. E. Valderrama, R. Ortega, and A. Stankovic, "Analysis and experimentation of nonlinear adaptive controllers for the series resonant converter," IEEE Trans. Power Electron., vol. 15, no. 3, pp. 536-544, May 2000.

[4] H.-S. Choi and B. H. Cho, "Novel zero-current-switching (ZCS) PWM switch cell minimizing additional conduction loss," IEEE Trans. On Industrial Electronics, Vol. 49, No. 1, P. 165-172, February 2002. 
[5] H.-S. Choi, J.-W. Kim, and B. H. Cho, "Novel zero-voltage and zerocurrent-switching (ZVZCS) full-bridge PWM converter using coupled output inductor," IEEE Trans. On Power Electronics, Vol. 17, No. 5, P. 641-648, September 2002.

[6] X. Ruan and Y. Yan, "A novel zero-voltage and zero-current-switching PWM full-bridge converter using two diodes in series with the lagging leg," IEEE Trans. On Industrial Electronics, Vol. 48, No. 4, P. 777-785, August 2001.

[7] J. M. Quero, J. M. Carrasco, and L. G. Franquelo, "Implementation of a neural controller for the series resonant converter," IEEE Trans. On Industrial Electronics, Vol. 49, No. 3, P. 628-639, June 2002.

[8] F. Kamran, R. G. Harley, B. Burton, T. G. Habetler, and M. A. Brooke, "A fast on-line neural-network training algorithm for a rectifier regulator," IEEE Trans. On Power Electronics, Vol. 13, No. 2, March 1998.

[9] B. Choi, J. Kim, B. H. Cho, S. Choi, and C. M. Wildrick, "Designing control loop for DC-to-DC converters loaded with unknown AC dynamics," IEEE Trans. On Industrial Electronics, Vol. 49, No. 4, P. 925-932, August 2002.

[10] Texas Instrument Inc, "BiCMOS advanced phase shift PWM controller," Data sheet of UCC3895, January 2001.

[11] B. Andreycak, "Designing a Phase Shifted Zero Voltage Transition (ZVT) Power Converter," High Frequency Power Conference Proceedings, 1992 